\title{
Observational evidence for the first stars and constraints on stellar models
}

\author{
Wako Aoki* \\ National Astronomical Observatory of Japan \\ E-mail: aoki.wako@nao.ac.jp
}

Recent progress in the search for metal-poor stars, including the discovery of a Ultra-Metal-Poor star $([\mathrm{Fe} / \mathrm{H}] \sim-5)$ with no excess of carbon in the Milky Way halo and Extremely Metal-Poor (EMP) stars $([\mathrm{Fe} / \mathrm{H}]<-3)$ in dwarf galaxies is reviewed. These discoveries indicate that lowmass stars have been formed at metallicities significantly lower than previously believed, possibly in small systems which may be observed as ultra-faint dwarf galaxies at present. The chemical compositions of EMP stars (in particular objects with $[\mathrm{Fe} / \mathrm{H}]<-3.5$ ), however, show complicated trends and/or scatter that is not simply explained by the above scenario. Statistics obtained for EMP stars, such as metallicity distribution functions, fractions of carbon-enhanced stars, and Li abundances, are discussed.

XII International Symposium on Nuclei in the Cosmos

August 5-12, 2012

Cairns, Australia

*Speaker. 


\section{Searches for the lowest metallicity stars in the Milky Way}

Elemental abundances of the chemically most primitive stars in the Milky Way and local group galaxies are believed to record the nucleosynthesis yields of the first stars in the universe. Searches for metal-poor stars are the basis of studies in this field.

In spite of the great efforts of searches for metal-free low-mass stars in the Galaxy, no object with $[\mathrm{Fe} / \mathrm{H}]<-4$ has been reported until the end of the 20th century. Existence of a metallicity limit around $[\mathrm{Fe} / \mathrm{H}]=-4$ was suggested from these results. The discovery of HE 0107-5240 which has $[\mathrm{Fe} / \mathrm{H}]=-5.2[1]$ has broken this limit. The review paper by Beers \& Christlieb [2] defines the nomenclature for stars with different metallicity, in which "Hyper Metal-Poor (HMP)" and "Ultra Metal-Poor (UMP)" are assigned for objects with $[\mathrm{Fe} / \mathrm{H}]<-5$ and $<-4$, respectively, while the range $-4<[\mathrm{Fe} / \mathrm{H}]<-3$ is called "Extremely Metal-Poor (EMP)".

The metallicity is usually estimated by the iron abundance $([\mathrm{Fe} / \mathrm{H}])$. The most iron-deficient star known to date is $\mathrm{HE} 1327-2326$ which has $[\mathrm{Fe} / \mathrm{H}]=-5.4[3,4]$. The $[\mathrm{Fe} / \mathrm{H}]$ value is slightly dependent on the analysis methods, i.e. including or neglecting the non-LTE effects and 3D effects of the stellar atmosphere. This object, as well as HE 0107-5240, however, has very large excesses of light elements $(\mathrm{C}, \mathrm{N}$ and $\mathrm{O}$ ) with respect to iron, in particular carbon ([C/Fe] $\sim 4)$. These light elements would play important roles in the cooling process of gas clouds and then in low-mass star formation. Including these elements in metallicity estimates, the existence of some critical metallicity for low-mass star formation was supported by the discovery of these carbon-enhanced stars.

The recent discovery of the new UMP star SDSS J1029+1729 ([Fe/H] -5, [5]) has a large impact on such discussion, because this object shows no excess of light elements $([\mathrm{C} / \mathrm{Fe}]<+1)$. The formation mechanism of low-mass stars at such low-metallicity is a hot topic in this field $[6,7]$.

Including HE 0557-4840 with $[\mathrm{Fe} / \mathrm{H}]=-4.8$ [8], which also shows an enhancement of carbon $([\mathrm{C} / \mathrm{Fe}]=+1.6)$, four UMP/HMP stars are currently known (there are a few more stars that could have metallicity slightly lower than $[\mathrm{Fe} / \mathrm{H}]=-4.0$ ). Two of the four stars are red giants, while the other two are main-sequence turn-off or subgiant stars. Three stars are carbon-rich, while the other shows no excess of light elements. We thus find diversity in the UMP/HMP sample even though the sample size is still very small. Further searches for UMP stars are clearly desired to understand the nature of this class of objects and low-mass star formation in the very early universe.

\section{Statistics of Extremely Metal-Poor Stars}

Chemical abundance studies for selected EMP stars in the past decade have provided very useful constraints on nucleosynthesis in early generations of stars, particularly in very massive stars and supernova explosions [9] and on neutron-capture processes [10]. On the other hand, in order to understand the roles of these processes in the chemical enrichment of the Milky Way and nearby galaxies, statistics of these objects is required. Thanks to progress of metal-poor star surveys and follow-up spectroscopy, the number of EMP stars studied based on high resolution spectra becomes larger than 200. Here observational facts found by statistics of EMP stars are reviewed and discussed. 


\subsection{Metallicity Distribution Function}

The metallicity distribution function (MDF) is an important indicator of the chemical evolution of the system. The MDF of the Milky Way halo has been studied by photometric and low- to medium-resolution spectroscopy $[11,12]$. Recently, the MDF in the low metallicity range $([\mathrm{Fe} / \mathrm{H}]<$ -2.5 ) has been studied based on medium-resolution spectroscopy for a large number of stars found by the Hamburg/ESO survey $[13,14]$. They determined the slope of the MDF in this metallicity range, and suggested a cut-off at $[\mathrm{Fe} / \mathrm{H}]=-3.5$.

The estimates of metallicity for the lowest metallicity are, however, not as certain as for less metal-poor stars. Our recent high-resolution studies of metal-poor stars found by the SDSS [15] demonstrate that there exist six objects with $[\mathrm{Fe} / \mathrm{H}]<-3.5$ among the 70 stars with $[\mathrm{Fe} / \mathrm{H}]<-3$, suggesting the existence of the low metallicity tail in the MDF. Similar results are obtained by the recent study based on the high resolution spectroscopy of Yong et al. [16].

\subsection{Carbon-enhanced stars}

A remarkable feature found in very metal-poor stars is that many objects show large excesses of carbon. Figure 1 shows carbon abundance ratios ([C/Fe]), as a function of $[\mathrm{Fe} / \mathrm{H}]$, for Milky Way field stars. The data are taken from the SAGA database [17][18], which contains abundance data based on high resolution spectroscopy reported in literature. Although the sample would not be complete, in particular in the less metal-poor ranges $([\mathrm{Fe} / \mathrm{H}]>-2.5)$, the high fraction of carbon-enhanced stars is obvious. Here objects with $[\mathrm{C} / \mathrm{Fe}]>+1.0$ (filled circles) are classified into Carbon-Enhanced Metal-Poor (CEMP) stars. Other definitions have been adopted by previous studies (e.g., $[\mathrm{C} / \mathrm{Fe}]>+0.7[19])$. However, there is a gap in the distribution of $[\mathrm{C} / \mathrm{Fe}]$ around $[\mathrm{C} / \mathrm{Fe}]=+0.7-+1.0$, and the classification is not very sensitive to the definition of CEMP stars. More systematic studies report that the fraction of CEMP is as high as $10-20 \%$, and could be higher in the lower metallicity range $([\mathrm{Fe} / \mathrm{H}]<-2.5)[20-22]$.

CEMP stars are separated into at least two subclasses. One is a group of objects that show large excesses of heavy neutron-capture elements (e.g., Ba). The excesses of carbon and heavy elements are interpreted as a result of mass transfer from a companion AGB star, which should already have evolved to an unseen white dwarf. The other subclass consists of CEMP-no stars that show no excess of heavy neutron-capture elements. The boundary of the two subclasses is set at $[\mathrm{Ba} / \mathrm{Fe}]=+0.5$ by Beers \& Christlieb [2].

Figure 2 shows the Ba abundance ratios as a function of $[\mathrm{Fe} / \mathrm{H}]$ for CEMP stars and non-CEMP stars. There are only several stars that have $[\mathrm{Ba} / \mathrm{Fe}]>+0.5$ among non-CEMP stars. The Ba-rich objects at $[\mathrm{Fe} / \mathrm{H}] \sim-3$ with no carbon-excess also show large excesses of Eu, and the enhancement of $\mathrm{Ba}$ is attributed to the r-process. On the other hand, 71 among 94 CEMP stars (76\%) show excesses of $\mathrm{Ba}$. This is comparable to the previous estimate of the fraction of CEMP-s stars among whole CEMP stars (70-80\% [19]). The fraction is, however, dependent on the metallicity. For $[\mathrm{Fe} / \mathrm{H}]<-3.0$, only 6 CEMP-s stars are found among $16 \mathrm{CEMP}$ stars (the fraction is $38 \%$ ). No CEMP-s star is found in $[\mathrm{Fe} / \mathrm{H}]<-3.3$. Hence, the CEMP-no subclass is dominant in the lowest metallicity range.

The origins of CEMP-no stars have not yet been identified. A promising candidate is the socalled faint supernova, which ejects only small amounts of $\mathrm{Fe}$ in the explosion, resulting in a very 


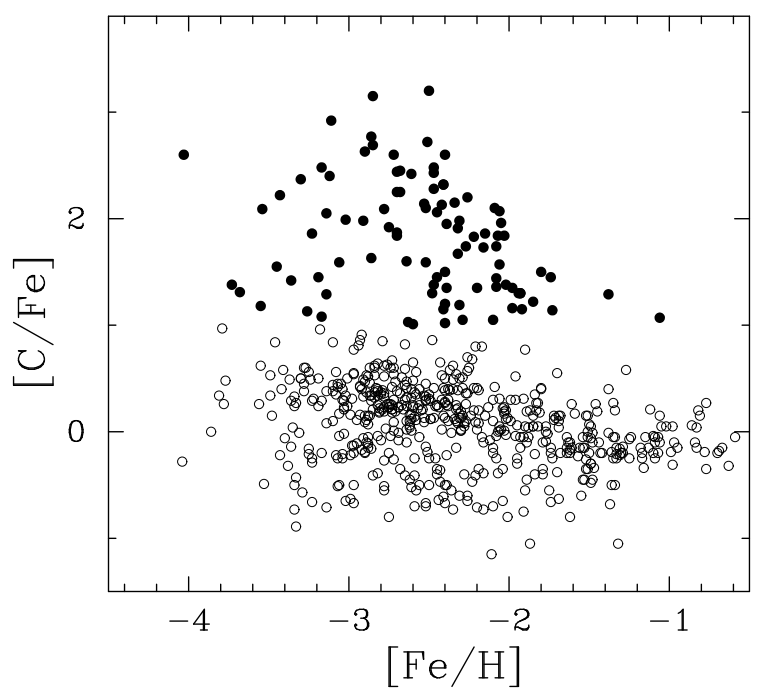

Figure 1: Carbon abundance ratio $([\mathrm{C} / \mathrm{Fe}])$ as a function of $[\mathrm{Fe} / \mathrm{H}]$. The data are taken from the SAGA database [18]. [C/Fe] $>+1.0$ (filled circles) are classified into Carbon-Enhanced Metal-Poor (CEMP) stars here.
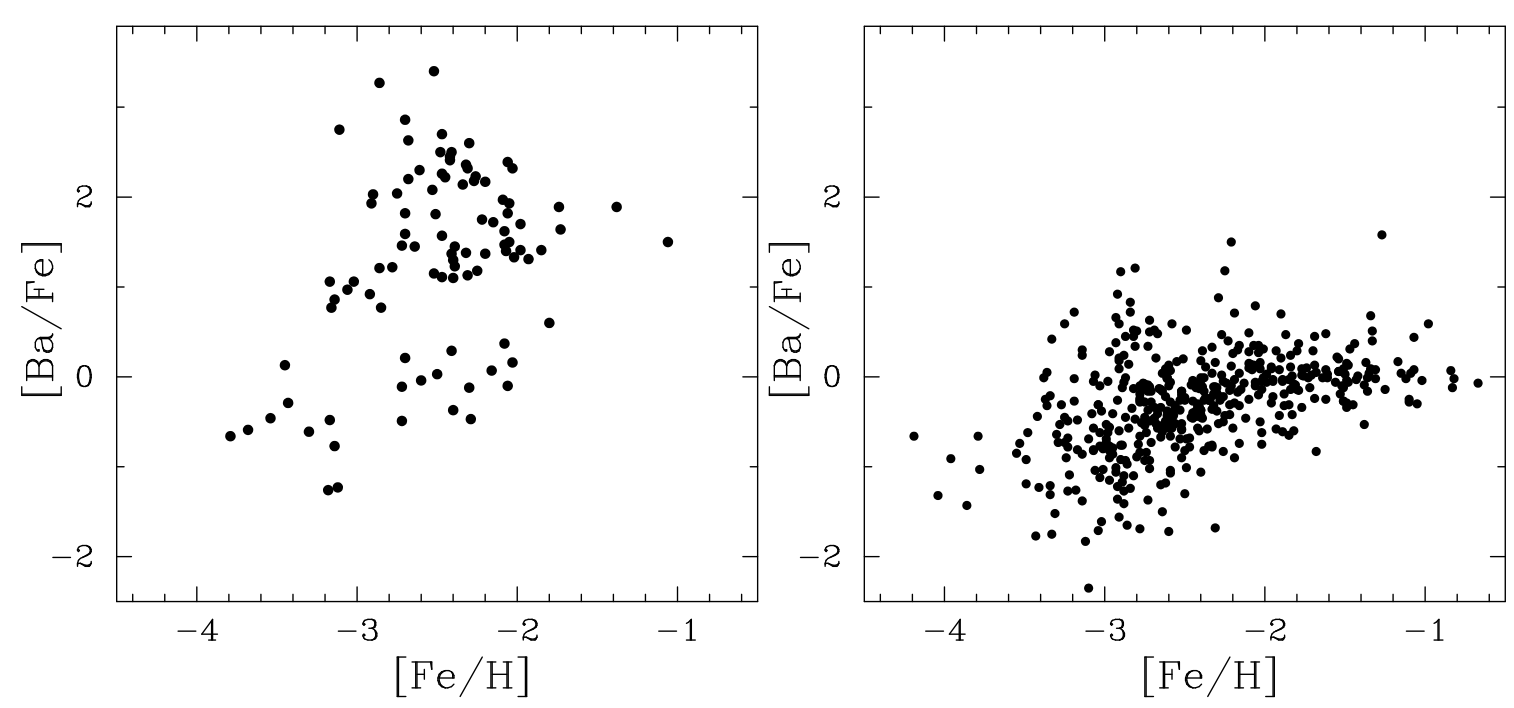

Figure 2: Ba abundance ratios of CEMP stars (left) and of objects for which carbon excess is not found (right). The data are taken from the SAGA database.

low abundance of $\mathrm{Fe}$ and a high $\mathrm{C} / \mathrm{Fe}$ ratio [9]. Such models explain well the abundance pattern of some CEMP-no stars [23]. The higher fraction of CEMP-no stars in the lower metallicity is a constraint on understanding the origins of this class of objects.

\subsection{Lithium}

$\mathrm{Li}$ abundances have been extensively studied for very metal-poor stars particularly to understand the reason for the serious discrepancy between the observed constant $\mathrm{Li}$ abundance, the so- 
called Spite plateau, of metal-poor turnoff stars $(A(\mathrm{Li})=\log (\mathrm{Li} / \mathrm{H})+12 \sim 2.2[24,25])$ and the primordial Li abundance estimated by standard Big Bang nucleosynthesis (BBN) models adopting the cosmological parameters determined from observations of the cosmic microwave background by the WMAP satellite $(A(\mathrm{Li})=2.72[26])$.

A hint for stellar intrinsic depletion, which could be a cause of the above discrepancy, is found in the $\mathrm{Li}$ abundances in EMP stars $([\mathrm{Fe} / \mathrm{H}]<-3)$. Recent studies [27-29] reported that the average $\mathrm{Li}$ abundance of EMP stars is lower than that of less metal-poor stars, and that significant star-tostar scatter exists. The strongest evidence for the depletion of $\mathrm{Li}$ in main-sequence turn-off EMP stars is the discrepancy of the Li abundances between the two components of the binary CS 22876$032([\mathrm{Fe} / \mathrm{H}] \sim-3.7)[30]$. Although the secondary is still a warm main-sequence star $\left(T_{\mathrm{eff}}\right)$ and no depletion of $\mathrm{Li}$ is expected by standard stellar evolution models, its $\mathrm{Li}$ abundance is 0.4 dex lower than that of the primary. If such depletion is effective in all metal-poor stars, that could be a cause of the discrepancy between the Li abundances predicted by the BBN model and the Spite plateau value.

On the other hand, no significant scatter is found for Li abundances in turn-off stars with $-3<[\mathrm{Fe} / \mathrm{H}]<-2$ [31]. This is also demonstrated by the measurement for the two components of the binary system G 166-45, which have similar stellar parameters of those of CS 22876-032 [32] except for the metallicity. Hence, further studies are required for quantitative estimates of the effect of the Li depletion inside metal-poor stars in general.

Another surprising fact is the non-detection of $\mathrm{Li}$ in the HMP star HE 1327-2326 (A(Li) $<0.6)$ [33] and the UMP star SDSS J1029+1729 $(A(\mathrm{Li})<1.1)$. These are main-sequence turn-off stars with $T_{\text {eff }}>5800 \mathrm{~K}$, in which surface convection is not expected to be so deep as Li is significantly depleted. These low Li abundances might be related to the Li depletion found in EMP stars, however, no promising scenario is proposed to explain consistently the Li abundances in the whole sample.

\subsection{Neutron-capture elements}

Elements heavier than $\mathrm{Zn}(Z=30)$ also provide useful constraints on understanding nucleosynthesis and evolution of early generations of stars. Sr and Ba have been extensively measured for EMP stars using the strong resonance doublet lines of their singly ionized species in the optical range (Sr II $4078 \AA$, $4215 \AA$ and Ba II $4554 \AA$, $4934 \AA$ ).

$\mathrm{Sr}$ and $\mathrm{Ba}$ represent the elements around the first and second abundance peaks of neutroncapture elements in solar system material corresponding to the neutron-magic numbers 50 and 82. Although the two elements are efficiently produced by the slow neutron-capture process (sprocess), the contribution of the s-process in (low-mass) AGB stars to EMP stars is expected to be small (or negligible) because of the long time-scale of the evolution of low-mass stars. Exceptions are carbon-enhanced objects with excess of s-process elements, which would have received material through mass transfer in binary systems.

The r-process (main r-process [34]) is believed to be the major contributor to heavy neutroncapture elements in EMP stars. However, measurements of over-all abundance patterns of neutroncapture elements for metal-poor stars have revealed the existence of other sources of light neutroncapture elements, including Sr. This is clearly found in the well-known bright metal-poor stars HD 122563 and HD 88609, in which large excesses of only light neutron-capture elements are 
found $[35,36]$. The process responsible for the formation of light neutron-capture elements is tentatively called the LEPP (Lighter Elements Primary Process [37]) or weak r-process [38].

The abundance distributions of Sr and Ba exhibit very large scatter in very metal-poor stars, even if carbon-enhanced objects are excluded from the sample. The scatter is as large as 3 dex at $[\mathrm{Fe} / \mathrm{H}] \sim-3$ both in $[\mathrm{Sr} / \mathrm{Fe}]$ and $[\mathrm{Ba} / \mathrm{Fe}]$ distributions. Interestingly, the scatter of the abundance ratios of the two elements ([Sr/Ba]) is also very large. According to the SAGA database [17], 30$40 \%$ of objects with $-3.5<[\mathrm{Fe} / \mathrm{H}]<-3.0$ have high $\mathrm{Sr} / \mathrm{Ba}$ ratios $([\mathrm{Sr} / \mathrm{Ba}]>+0.5)$. This suggests that the progenitors of EMP stars in this metallicity range (presumably very massive stars) are the sources of the excess of light neutron-capture elements.

By contrast, no Sr-enhanced star is found in $[\mathrm{Fe} / \mathrm{H}]<-3.5$, although the sample size of objects for which both $\mathrm{Sr}$ and $\mathrm{Ba}$ are detected in this metallicity range is still small. This indicates that the progenitors of $[\mathrm{Fe} / \mathrm{H}]<-3.5$ stars are significantly different from those of $[\mathrm{Fe} / \mathrm{H}]>-3.5$ stars. The details of the abundance distributions and implications are discussed by Aoki [39].

\section{Extremely Metal-Poor stars in dwarf galaxies}

Another important progress is that a number of EMP stars have been discovered in dwarf galaxies around the Milky Way. Our local group of galaxies consists of the two large spiral galaxies the Milky Way and M31, and other smaller galaxies. A few tens dwarf galaxies have been found around the Milky Way, and the number is increasing by the recent discoveries of the so-called ultra faint dwarf galaxies (see below).

Until several years ago, no EMP stars have been found in dwarf galaxies, and some difference in the metallicity distribution function between dwarf galaxies and the field halo was suggested based on the metallicity estimates from medium resolution spectroscopy (e.g. [40]). However, subsequent studies adopting new calibrations for the metallicity estimates, which became available by comparisons with estimates based on high resolution spectra for a significant number of EMP stars, have revealed that EMP stars are not deficient in dwarf galaxies [41]. The results are confirmed by the high resolution follow-up studies, which have already found several objects with $[\mathrm{Fe} / \mathrm{H}]<-3.5$ in dwarf galaxies [42].

In particular, the average metallicity of ultra faint dwarf galaxies is quite low, and the fraction of EMP stars among the sample seems to be quite high [43]. These galaxies have only $\sim 10^{4}$ solar luminosities, indicating that the number of stars involved in a galaxy is very small. These galaxies have been discovered only recently by SDSS [44] because of their low stellar density.

The chemical compositions of EMP stars in dwarf galaxies studied by high resolution spectroscopy are similar to those of EMP stars in the field halo [42, 45], while significant differences are found for less metal-poor stars [46]. Discoveries of EMP stars in (ultra faint) dwarf galaxies suggest that the early generations of stars have been formed in small stellar systems like the current (ultra faint) dwarf galaxies and incorporated into the Milky Way halo. The number of EMP stars studied for dwarf galaxies is, however, still too small to derive any conclusion, and further observational studies are strongly desired. 


\section{Future prospect}

The recent studies of the most metal-poor stars revealed that low-mass stars can be formed even at $[\mathrm{Fe} / \mathrm{H}]<<-4$. Such studies encourage further searches for more metal-poor, or even metal-free low-mass stars in the Galaxy. Another important question is the existence of super massive stars among first generations of stars that ended their lives by pair-instability supernovae. Large scale surveys of Galactic stars with multi-object spectrograph like SDSS, LAMOST and the photometric survey Skymapper will play important roles in the searches for first generations of stars or their signature.

For the follow-up high resolution spectroscopy for these field stars, as well as for candidate EMP stars in dwarf galaxies, high resolution spectrographs mounted on larger telescopes are required. That will be available by next generation Extremely Large Telescopes (ELTs) planned for operation in 2020s, including Thirty Meter Telescope (TMT), Giant Magellan Telescope (GMT) and European ELT (E-ELT).

\section{Discussion}

Weiping Lin: Is scattering in $\mathrm{Li}$ and $\mathrm{Ba}$ abundance meaning one need to use more parameter to normalise the data in order to find new physics? - Aoki: Although the size of the scatter in Li and $\mathrm{Ba}$, and probably the reason for them, is different, such scatter is a key to understanding the phenomena found in EMP stars.

Yeisson Osorio:Atomic diffusion was proposed as possible explanation of the cosmological Li problem. Is it as relation diffusion- $\mathrm{Z}$ that help to explain the scatter of Li in EMP stars? - Aoki: Metallicity dependence is necessary to explain the trend and scatter of $\mathrm{Li}$ abundances of metalpoor stars. It may be difficult, however, to explain the extremely low $\mathrm{Li}$ abundances in the two UMP/HMP stars even if metallicity dependence is included in the models of diffusion.

Raphael Hirschi: Is the absence of Sr-enriched stars at the lowest metallicities statistically significant? - Aoki: There are only four stars for which $\mathrm{Sr} / \mathrm{Ba}$ ratio is determined in $[\mathrm{Fe} / \mathrm{H}]<-3.6$, and the statistics are still insufficient. There are, however, several stars for which low $\mathrm{Sr}$ abundance or its low upper limit is determined without $\mathrm{Ba}$ abundance measurement, supporting the suggestion for the low $\mathrm{Sr} / \mathrm{Ba}$ in this metallicity range.

Chiaki Kobayashi: Can you summarise the statistics of binarity of $\mathrm{C} \& \mathrm{Ba}$ enhanced stars? - Aoki: High frequency of the binaries among CEMP stars with Ba-excess is found by previous studies as well as by our current monitoring of radial velocities for these stars. There are, however, several stars in this class that do not show any change of radial velocity, and further monitoring is meaningful.

W.A. was supported by the JSPS Grants-in-Aid for Scientific Research (23224004).

\section{References}

[1] N. Christlieb, M. S. Bessell, T. C. Beers, B. Gustafsson, A. Korn, P. S. Barklem, T. Karlsson, M. Mizuno-Wiedner, and S. Rossi, A stellar relic from the early Milky Way, Nature 419 (904) 2002, [arXiv:astro-ph/0211274] 
[2] T. C. Beers, and N. Christlieb, The Discovery and Analysis of Very Metal-Poor Stars in the Galaxy, Ann. Rev. Astron. Astrophys. 43 (531) 2005

[3] A. Frebel, W. Aoki, N. Christlieb, H. Ando, M. Asplund, P. S. Barklem, T. C. Beers, K. Eriksson, C. Fechner, M. Y. Fujimoto, S. Honda, T. Kajino, T. Minezaki, K. Nomoto, J. E. Norris, S. G. Ryan, M. Takada-Hidai, S. Tsangarides, and Y. Yoshii, Nucleosynthetic signatures of the first stars, Nature 434 (871) 2005, [arXiv:astro-ph/0503021]

[4] W. Aoki, A. Frebel, N. Christlieb, J. E. Norris, T. C. Beers, T. Minezaki, P. S. Barklem, S. Honda, M. Takada-Hidai, M. Asplund, S. G. Ryan, S. Tsangarides, K. Eriksson, A. Steinhauer, C. P. Deliyannis, K. Nomoto, M. Y. Fujimoto, H. Ando, Y. Yoshii, and T. Kajino, HE 1327-2326, an Unevolved Star with $[\mathrm{Fe} / \mathrm{H}]<-5.0$. I. A Comprehensive Abundance Analysis, Astrophys. J. 639 (897) 2006, [arXiv:astro-ph/0509206]

[5] E. Caffau, P. Bonifacio, P. François, L. Sbordone, L. Monaco, M. Spite, F. Spite, H.-G. Ludwig, R. Cayrel, S. Zaggia, F. Hammer, S. Randich, P. Molaro, and V. Hill, An extremely primitive star in the Galactic halo, Nature 477 (67) 2011, [arXiv:astro-ph/1203.2612]

[6] R. Schneider, K. Omukai, M. Limongi et al. The formation of the extremely primitive star SDSS J102915+172927 relies on dust, Mon. Not. Roy. Astron. Soc. 423 (L60) 2012

[7] T. Nozawa, T. Kozasa \& K. Nomoto, Can the Growth of Dust Grains in Low-metallicity Star-forming Clouds Affect the Formation of Metal-poor Low-mass Stars? Astrophys. J. Lett. 756 (L35) 2012

[8] J. E. Norris, N. Christlieb, A. J. Korn, K. Eriksson, M. S. Bessell, T. C. Beers, L. Wisotzki, and D. Reimers, HE 0557-4840: Ultra-Metal-Poor and Carbon-Rich, Astrophys. J. 670, (774) 2007, [astro-ph/0707.2657]

[9] N. Tominaga, H. Umeda, \& K. Nomoto, Supernova Nucleosynthesis in Population III 13-50 Msolar Stars and Abundance Patterns of Extremely Metal-poor Stars, Astropys. J. 660 (516), 2007

[10] C. Sneden \& J. J. Cowan, Genesis of the Heaviest Elements in the Milky Way Galaxy, Science 299 (70) 2003

[11] S. G. Ryan \& J. E. Norris Subdwarf Studies. III. The Halo Metallicity Distribution Astron. J. 101 (1865) 1991

[12] B. W. Carney, D. W. Latham, J. B. Laird \& L. A. Aguilar, A survey of proper motion stars. 12: an expanded sample, Astron. J. 107 (2240) 1994

[13] T. Schörck, N. Christlieb, J. G. Cohen et al. The stellar content of the Hamburg/ESO survey. V. The metallicity distribution function of the Galactic halo Astron. Astrophys. 507 (817) 2009

[14] H. N. Li, N. Christlieb, T. Schörck et al., The stellar content of the Hamburg/ESO survey. VI. Metallicity distribution of main-sequence turnoff stars in the Galactic halo, Astron. Astrophys. 521 (A10) 2010

[15] W. Aoki, T. C. Beers, Y. S. Lee, et al., High-Resolution Spectroscopy of Extremely Metal-Poor Stars from SDSS/SEGUE: I. Atmospheric Parameters and Chemical Compositions, Astron. J. in press, [arXiv:1210.1946]

[16] D. Yong, J. E. Norris, M. S. Bessell et al., The Most Metal-Poor Stars. III. The Metallicity Distribution Function and CEMP Fraction, Astrophys. J. in press, [arXiv:1208.3016]

[17] T. Suda, Y. Katsuta, S. Yamada, et al., Stellar Abundances for the Galactic Archeology (SAGA) Database - Compilation of the Characteristics of Known Extremely Metal-Poor Stars, Publ. Astron. Soc. Japan 60 (1159) 2008 
[18] T. Suda, this volume

[19] W. Aoki, T. C. Beers, N. Christlieb, et al., Carbon-enhanced Metal-poor Stars. I. Chemical Compositions of 26 Stars, Astrophys. J. 655 (492) 2007

[20] S. Lucatello, S., T. C. Beers, N. Christlieb, et al., The Frequency of Carbon-enhanced Metal-poor Stars in the Galaxy from the HERES Sample, Astrophys. J. Lett. 652 (L37) 2006

[21] A. Frebel, N. Christlieb, J. E. Norris, et al., Bright Metal-poor Stars from the Hamburg/ESO Survey. I. Selection and Follow-up Observations from 329 Fields, Astrophys. J. 652 (1585) 2006

[22] D. Carollo, T. C. Beers, J. Bovy, et al., Carbon-enhanced Metal-poor Stars in the Inner and Outer Halo Components of the Milky Way, Astrophys. J. 744 (195) 2012

[23] H. Ito, W. Aoki, S. Honda \& T. C. Beers, BD+44 493: A Ninth Magnitude Messenger from the Early Universe; Carbon Enhanced and Beryllium Poor, Astrophys. J. Lett. 698 (L37) 2009

[24] M. Spite, \& F. Spite, Lithium abundance at the formation of the Galaxy, Nature 297 (483) 1982

[25] S. G. Ryan, J. E. Norris \& T. C. Beers, The Spite Lithium Plateau: Ultrathin but Postprimordial, Astrophys. J. 523 (654) 1999

[26] R. H. Cyburt, B. D. Fields \& K. A. Olive, An update on the big bang nucleosynthesis prediction for 7Li: the problem worsens, J. Cosm. and Astropart. Phys. 11 (12) 2008

[27] P. Bonifacio, P. Molaro, T. Sivarani, et al., First stars VII - Lithium in extremely metal poor dwarfs, Astron. Astrophys. 462 (851) 2007

[28] W. Aoki, P. S. Barklem, T. C. Beers, et al., Lithium Abundances of Extremely Metal-Poor Turnoff Stars, Astrophys. J., 698 (1803) 2009

[29] L. Sbordone, P. Bonifacio, E. Caffau et al., The metal-poor end of the Spite plateau. I. Stellar parameters, metallicities, and lithium abundances, Astron. Astrophys. 522 (A26) 2010

[30] J. I. González Hernández, P. Bonifacio, H/-G. Ludwig et al., First stars XI. Chemical composition of the extremely metal-poor dwarfs in the binary CS 22876-032, Astron. Astrophys. 480 (223) 2008

[31] J. Meléndez, L. Casagrande, I. Ramírez, M. Asplund, \& W. J. Schuster, Observational evidence for a broken Li Spite plateau and mass-dependent Li depletion, Astron. Astrophys. 515 (L3) 2010

[32] W. Aoki, H. Ito, \& A. Tajitsu, Examination of the Mass-dependent Li Depletion Hypothesis by the Li Abundances of the Very Metal-poor Double-lined Spectroscopic Binary G166-45, Astrophys. J. Lett. 751 (L6) 2012

[33] A. Frebel, R. Collet, K. Eriksson, N. Christlieb \& W. Aoki, HE 1327-2326, an Unevolved Star with $[\mathrm{Fe} / \mathrm{H}]<-5.0$. II. New 3D-1D Corrected Abundances from a Very Large Telescope UVES Spectrum Astrophys. J. 684 (588) 2008

[34] J. W. Truran, J. J. Cowan, C. A. Pilachowski, \& C. Sneden, Probing the Neutron-Capture Nucleosynthesis History of Galactic Matter, Publ. Astron. Soc. Pacific 114 (1293) 2002

[35] S. Honda, W. Aoki, Y. Ishimaru, S. Wanajo \& S. G. Ryan, Neutron-Capture Elements in the Very Metal Poor Star HD 122563 Astrophys. J. 643 (1180) 2006

[36] S. Honda, W. Aoki, Y. Ishimaru, \& S. Wanajo, Neutron-Capture Elements in the Very Metal-poor Star HD 88609: Another Star with Excesses of Light Neutron-Capture Elements, Astrophys. J. 666 (1189) 2007 
[37] C. Travaglio, R. Gallino, E. Arnone, et al., Galactic Evolution of Sr, Y, And Zr: A Multiplicity of Nucleosynthetic Processes Astrophys. J. 601 (864) 2004

[38] S. Wanajo, \& Y. Ishimaru, r-process calculations and Galactic chemical evolution Nuclear Physics A 777 (676) 2006

[39] W. Aoki, Neutron-Capture Elements in Extremely Metal-Poor Stars, AIP conf. Ser., Proc. of Origin of Matter and Evolution of Galaxies in press

[40] A. Helmi, M. J. Irwin, \& E. Tolstoy, et al., A New View of the Dwarf Spheroidal Satellites of the Milky Way from VLT FLAMES: Where Are the Very Metal-poor Stars?, Astrophys. J. Letters 651 (L121) 2006

[41] E. Starkenburg, V. Hill, E. Tolstoy et al., The NIR Ca ii triplet at low metallicity. Searching for extremely low-metallicity stars in classical dwarf galaxies, Astron. Astrophys. 513 (A34) 2010

[42] A. Frebel, E. N. Kirby, \& J.D. Simon, Linking dwarf galaxies to halo building blocks with the most metal-poor star in Sculptor, Nature 464 (72) 2010

[43] E. N. Kirby, J. D. Simon, M. Geha, P. Guhathakurta \& A. Frebel, Uncovering Extremely Metal-Poor Stars in the Milky Way's Ultrafaint Dwarf Spheroidal Satellite Galaxies, Astrophys. J. Lett. 685 (L43) 2008

[44] V. Belokurov, D. B. Zucker, N. W. Evans et al., Cats and Dogs, Hair and a Hero: A Quintet of New Milky Way Companions, Astrophys. J. 654 (897) 2007

[45] J. E. Norris, G. Gilmore, R. F. Wyse, D. Yong \& A. Frebel, An Extremely Carbon-rich, Extremely Metal-poor Star in the Segue 1 System, Astrophys. J. Letters 722 (L104) 2010

[46] E. Tolstoy, V. Hill, \& M. Tosi Star-Formation Histories, Abundances, and Kinematics of Dwarf Galaxies in the Local Group, Ann. Rev. Astron. Astrophys. 47 (371) 2009 